Check for updates

Cite this: RSC Adv., 2019, 9, 4514

Received 4th January 2019

Accepted 25th January 2019

DOI: $10.1039 / c 9 r a 00075 e$

rsc.li/rsc-advances

\section{The study of the characteristics and hydrolysis properties of naringinase immobilized by porous silica material}

\author{
Jian Luo, ${ }^{a}$ Qian Li, (D) ${ }^{* a}$ Xitong Sun, ${ }^{* b}$ Jing Tian, ${ }^{a}$ Xu Fei, (D) ${ }^{a}$ Feng Shi, ${ }^{a}$ Nan Zhang ${ }^{a}$ \\ and Xiangli Liu ${ }^{a}$
}

Silica material has high specific surface area and excellent chemical stability, which make it useful for enzyme immobilization. In this work, naringinase was immobilized from fermentation broth of Aspergillus niger FFCC uv-11 by silica materials with different pore diameters of $2 \mathrm{~nm}$ (MCM-41), $7.7 \mathrm{~nm}$ (SBA-15) and $80 \mathrm{~nm}$ (silica gel). It was shown that SBA-15 had the highest naringinase activity, and this was chosen as a suitable carrier material for naringinase immobilization. First, SBA-15 was modified by glutaraldehyde at a concentration of $7 \%$ at $25^{\circ} \mathrm{C}$ for $2 \mathrm{~h}$, and it was then used for the immobilization of naringinase. At pH 3.5, the immobilized naringinase activity reached $467.62 \mathrm{U} \mathrm{g}^{-1}$ at $40{ }^{\circ} \mathrm{C}$ for $4 \mathrm{~h}$ when the initial naringinase activity was $89.04 \mathrm{U} \mathrm{mL}^{-1}$. Furthermore, at the optimal reaction temperature of $45^{\circ} \mathrm{C}$ and $\mathrm{pH}$ of 4.5 , the binding efficiency, activity recovery rate and specific activity of the immobilized naringinase were $63.66 \%, 87.64 \%$ and $517.43 \mathrm{U} \mathrm{g}^{-1}$, respectively. Compared with free naringinase, in naringin hydrolysis, the immobilized naringinase performed over a wide $\mathrm{pH}$ application range and had good thermal stability. Even more important, the immobilized naringinase retained $61.81 \%$ of the residual naringinase activity after eight consecutive cycles, and kept $80.95 \%$ of the residual naringinase activity after one month of storage. This study provides an ideal carrier material and some basic data for naringinase immobilization technology, which will greatly promote the application of naringinase in industrial fruit juice processing.

\section{Introduction}

Naringin, the main bitter substance in citrus fruits, is a flavonoid that seriously affects the taste and flavor of fruit juice. ${ }^{1}$ To achieve debittering, in some juice product processing, free naringinase is used to completely hydrolyze naringin to naringenin and glucose, which can greatly reduce the bitterness value of the fruit juice..$^{2-4}$ As far as we know, naringinase is a complex enzyme composed of $\alpha$-L-rhamnosidase and $\beta$-Dglucosidase, and the hydrolysis of naringin has two steps. In the first step, $\alpha$-L-rhamnosidase hydrolyzes naringin into prunin (whose bitterness value is only one-third that of naringin) and rhamnose. ${ }^{5}$ In the second step, prunin is hydrolyzed into naringenin and glucose by $\beta$-D-glucosidase (Scheme 1). Additionally, prunin and rhamnose have some functions such as antiinflammatory, anti-cancer, anti-bacterial, anti-oxidation and anti-viral activity and cholesterol lowering.

However, considering the high price of naringinase and the difficulty of recovering free naringinase from fruit juice,

${ }^{a}$ Department of Bioengineering, Dalian Polytechnic University, 116034, Dalian, China. E-mail: liqian19820903@163.com

${ }^{b}$ Dalian Institute of Chemical Physics, Chinese Academy of Sciences, 116023, Dalian, China.E-mail:sunxt@dicp.ac.cn immobilized naringinase is welcomed owing to its high stability and ease of recycling. In addition, immobilized naringinase has excellent capacity for reuse, which can reduce the industrial process cost. ${ }^{6}$ Porous silica material is a good choice for naringinase immobilization, because it has ordered nanopore structure, large pore volume, appropriate pore size distribution, high thermal stability and chemical stability. More importantly, the surface of the mesoporous material is rich in active hydroxyl groups, which could be easily functionalized to change the surface properties. ${ }^{7}$ In this work, porous silica materials with different pore sizes ( $2 \mathrm{~nm}$ (MCM-41), $7.7 \mathrm{~nm}$ (SBA-15), $80 \mathrm{~nm}$ (silica gel)) were used as the carrier for naringinase immobilization. The materials were first silanized so that they possessed enough amino-groups, and then modified by glutaraldehyde for naringinase immobilization. ${ }^{8}$ SBA- 15 with an average pore size of $7.7 \mathrm{~nm}$ was found to have the highest naringinase activity, and so the characteristics and hydrolytic properties of the naringinase immobilized on this material were further studied.

\section{Materials and methods}

\subsection{Materials}

Aspergillus niger FFCC uv-11 was supplied by the Dalian Institute of Technology Culture Collection, ${ }^{9}$ and naringinase 


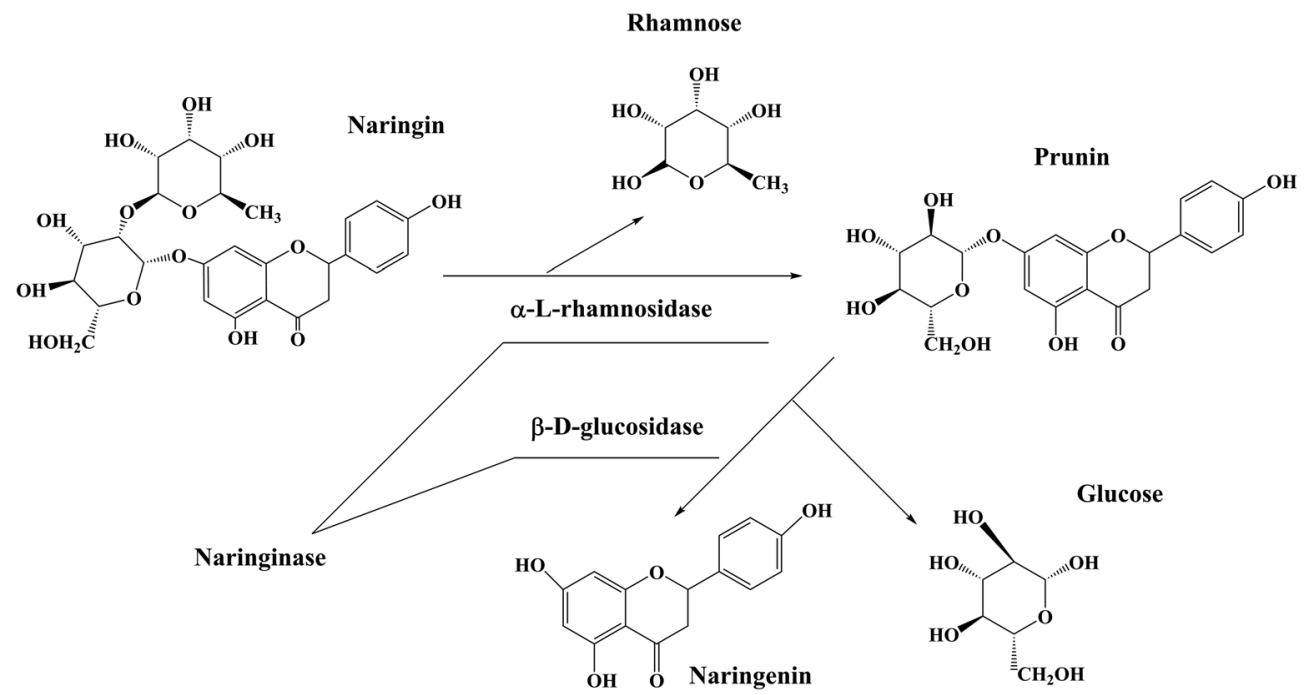

Scheme 1 Hydrolysis reaction process of naringinase.

fermentation broth was produced via this strain, with a naringinase activity of $838.22 \mathrm{U} \mathrm{mL}^{-1}$. Naringin (mass fraction $\geq$ 98\%) was purchased from Baoji Fang Sheng Biological development Co., Ltd, China. Chitosan (deacetylation 80-95\%), poly(ethylene oxide)-poly(propylene oxide)-poly(ethylene oxide) triblock copolymer Pluronic P123 $\left(\mathrm{EO}_{20} \mathrm{PO}_{70} \mathrm{EO}_{20}, M_{\mathrm{w}}=5800\right)$, tetraethyl orthosilicate (TEOS), cetyltrimethylammonium bromide (CTAB), sodium hydroxide, anhydrous ethanol, citric acid, disodium hydrogen phosphate, diethylene glycol, glutaraldehyde ( $50 \%$ by mass), toluene and dichloromethane were purchased from Sinopharm Chemical Reagent Co., Ltd and 3aminopropyl trimethoxysilane (APTMS) was supplied by Gelest Inc.

\subsection{Preparation of MCM-41}

MCM-41 was synthesized according to a previously reported method..$^{10-12}$ Briefly, $0.85 \mathrm{~g}$ of CTAB was completely dissolved in a mixture of $200 \mathrm{~g}$ of water and $14.3 \mathrm{~g}$ of aqueous ammonia solution. Subsequently, $4.37 \mathrm{~g}$ of TEOS was added to the above mixture with stirring. After 10 minutes, the mixture was kept at $80^{\circ} \mathrm{C}$ for $2 \mathrm{~h}$. Then it was filtered and washed with ethanol and water until neutrality, and then dried at $120{ }^{\circ} \mathrm{C}$. Last, the assynthesized product was calcined in air for $6 \mathrm{~h}$ at $550{ }^{\circ} \mathrm{C}$ and the MCM- 41 was obtained.

\subsection{Preparation of SBA-15}

An amount of $4.0 \mathrm{~g}$ of P123 was dissolved in a mixture of $30 \mathrm{~mL}$ of water and $120 \mathrm{~mL}$ of $2 \mathrm{~mol} \mathrm{~L}^{-1}$ hydrochloric acid. The mixture was stirred in a water bath at $35^{\circ} \mathrm{C}$, and $2.15 \mathrm{~g}$ of TEOS was then added. After stirring for $20 \mathrm{~h}$, the white emulsion was placed in a container and dried in an oven at $80{ }^{\circ} \mathrm{C}$ for 2 days. Next, it was suction filtered and washed with ethanol and water until neutrality and dried at $50^{\circ} \mathrm{C}$ for $24 \mathrm{~h}$. The filtrate was then calcined in a muffle furnace at $550{ }^{\circ} \mathrm{C}$ for $6 \mathrm{~h}$, and the end product was SBA-15..$^{13,14}$

\subsection{Silanization of immobilization material}

Twenty grams of dried immobilization material was put in a $250 \mathrm{~mL}$ round bottom flask, and then $110 \mathrm{~mL}$ of anhydrous toluene and $15 \mathrm{~mL}$ APTMS were added and the mixture was stirred under reflux at $110{ }^{\circ} \mathrm{C}$ for $24 \mathrm{~h}$ under nitrogen atmosphere. The product was filtered and washed successively with anhydrous toluene, dichloromethane, methanol, water and methanol, and dried at $80{ }^{\circ} \mathrm{C}$ overnight. ${ }^{15-17}$

\subsection{Aldehyde modification of immobilization material}

The silanized immobilization material was weighed and mixed with the diluted glutaraldehyde solution. ${ }^{18,19}$ After shaking for a certain time, the supernatant was centrifuged, and then washed three times with deionized water. After that, it was dried under vacuum at room temperature for later use.

\subsection{Preparation of immobilized naringinase}

A certain amount of modified material was mixed with naringinase fermentation broth, and citric acid buffer was added to adjust the $\mathrm{pH}$ value to 4.5 . Additionally, the mixture was shaken for $2 \mathrm{~h}$ at constant temperature, and then refrigerated at $4{ }^{\circ} \mathrm{C}$ for $4 \mathrm{~h}$. After centrifugation and washing, the immobilized naringinase was dried by vacuum at room temperature for later use.

\subsection{Determination of naringinase activity}

The naringinase activity was determined by the modified Davis method: ${ }^{20} 0.8 \mathrm{~mL}$ of $0.8 \mathrm{~g} \mathrm{~L}^{-1}$ naringin solution with $\mathrm{pH}$ of 4.5 was added into a flask containing $30 \mathrm{mg}$ of immobilized naringinase or $0.2 \mathrm{~mL}$ of free naringinase, and the flask was then placed in a water bath at $50{ }^{\circ} \mathrm{C}$ for 30 minutes. Then, $0.1 \mathrm{~mL}$ of naringinase reaction solution was added into $5 \mathrm{~mL}$ of diethylene glycol (volume fraction 90\%) and $0.1 \mathrm{~mL}$ of $4 \mathrm{~mol} \mathrm{~L}^{-1}$ $\mathrm{NaOH}$ solution. The mixture was left to stand for $15 \mathrm{~min}$ and then the absorbance of the solution was measured at $420 \mathrm{~nm}$. 
Definition of naringinase activity (U): the amount of naringinase required to degrade $1 \mu \mathrm{g}$ of naringin per minute at $\mathrm{pH}$ 4.5 and $45{ }^{\circ} \mathrm{C}$ is one unit of naringinase activity. Definition of the specific activity of naringinase $\left(\mathrm{U} \mathrm{g}^{-1}\right.$ or $\left.\mathrm{U} \mathrm{mL}^{-1}\right)$ : naringinase activity demonstrated by $1 \mathrm{~g}$ immobilized naringinase (or $1 \mathrm{~mL}$ free naringinase) at $\mathrm{pH} 4.5$ and $45^{\circ} \mathrm{C}$.

The experiments were carried out in triplicate.

\subsection{Calculation of naringinase-carrying rate and naringinase activity recovery}

Immobilized naringinase-carrying rate $(B)$ was calculated by the following equation: ${ }^{21}$

$$
B \%=\left(A_{0}-A_{1}\right) / A_{0} \times 100 \%
$$

wherein, $B$ represents the naringinase carrying rate, $A_{0}$ refers to the total naringinase activity in the naringinase solution before immobilization, and $A_{1}$ refers to the total naringinase activity remaining in the supernatant after immobilization.

Immobilized naringinase activity recovery $(R)$ was calculated by the following equation: ${ }^{21}$

$$
R \%=A_{2} /\left(A_{0}-A_{1}\right) \times 100 \%
$$

wherein, $R$ represents the recovery rate of the naringinase, $A_{0}$ and $A_{1}$ are the same as in eqn (1), and $A_{2}$ refers to the total activity of immobilized naringinase in the enzymatic reaction that was used to measure total naringinase activity.

Relative naringinase activity $(Q)$ was calculated by the following equation:

$$
Q \%=P / K \times 100 \%
$$

wherein, $Q$ represents the relative enzyme activity, $P$ refers to the enzyme activity under certain conditions, and $K$ refers to the highest enzyme activity under the same conditions.

\subsection{Characterization of materials}

A Spectrum 10 spectrophotometer (PerkinElmer, USA) was used to record the Fourier transform infrared (FT-IR) spectra of SBA15 and silanized SBA-15 between 400 and $4000 \mathrm{~cm}^{-1}$ with a resolution of $2 \mathrm{~cm}^{-1}$. The nitrogen adsorption/desorption isotherms were measured using a QuantaChrome Quadrasorb SI analyzer (USA) after vacuum degassing at $120^{\circ} \mathrm{C}$ for $6 \mathrm{~h}$. The specific surface areas and the pore volumes were calculated using the Brunauer-Emmett-Teller (BET) method.

\section{Results and discussion}

\subsection{Immobilized naringinase activity of materials with different pore sizes}

Naringinase was immobilized by silica materials with different pore sizes. It is indicated in Fig. 1 that before silanization and modification, the immobilized naringinase activities of the silicon materials with $2 \mathrm{~nm}, 7.7 \mathrm{~nm}$ and $80 \mathrm{~nm}$ pore size were $44.11,251.34$ and $228 \mathrm{U} \mathrm{g}^{-1}$, respectively, while after silanization and modification, the immobilized naringinase activities of

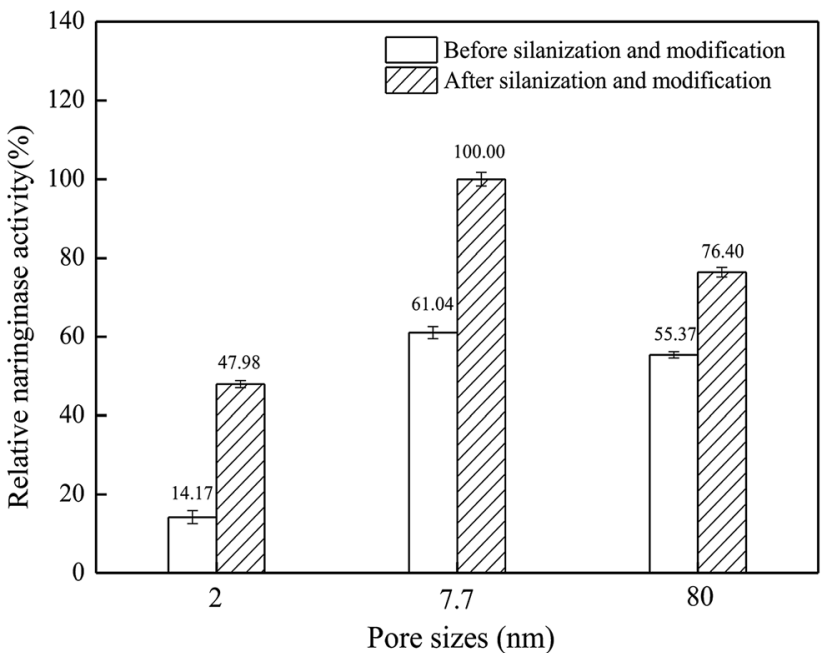

Fig. 1 Naringinase activity on silica materials with different pore sizes (the experiments were carried out in triplicate).

those materials were $149.35,411.76$ and $314.59 \mathrm{U} \mathrm{g}^{-1}$, respectively. SBA-15, with a pore size of $7.7 \mathrm{~nm}$, had the highest naringinase activity, and it was chosen for further study.

\subsection{Modification and characterization of immobilization material}

3.2.1 Immobilization material modification. Fig. 2 shows the effects of modification concentration, time and temperature on the immobilized naringinase activity. Fig. 2(A) shows that, as the glutaraldehyde concentration was gradually increased from $5 \%$ to $7 \%$, the naringinase activity gradually increased, and reached its maximum value at the glutaraldehyde concentration of $7 \%$. Glutaraldehyde concentrations lower than $7 \%$ might not supply enough binding sites for naringinase, which would induce lower naringinase activity. When the glutaraldehyde concentration was further increased from $7 \%$ to $9 \%$, the naringinase activity was not further enhanced, which might be because the presence of too many aldehyde groups could block entry of the naringinase into the pores of the material.

The effect of modification time on the immobilized naringinase activity is shown in Fig. 2(B). The optimum modification time was $5 \mathrm{~h}$, and glutaraldehyde required sufficient time to bind on SBA-15. In addition, for modification times longer than $5 \mathrm{~h}$, the immobilized naringinase activity tended to be stable. As shown in Fig. 2(C), $35{ }^{\circ} \mathrm{C}$ was the optimum modification temperature, which was suitable for the reaction between amino-group and glutaraldehyde.

3.2.2 Characterization. As shown in Fig. 3, both before and after silanization the characteristic peaks of SBA-15, 1088 and $3418 \mathrm{~cm}^{-1}$, were present in the FT-IR spectra of the materials, corresponding to $\mathrm{Si}-\mathrm{OH}$ (stretching vibration) and $\mathrm{Si}-\mathrm{O}-\mathrm{Si}$ bonds. More importantly, after silanization, there was a new peak at $880 \mathrm{~cm}^{-1}$, which was due to the bending vibration of $\mathrm{Si}-$ $\mathrm{OH}$ bonds. Furthermore, vibration of the $\mathrm{N}-\mathrm{H}$ bond appeared at $1512 \mathrm{~cm}^{-1}$, and the peaks due to the $\mathrm{C}-\mathrm{H}$ bond at $2973 \mathrm{~cm}^{-1}$ were sharply enhanced, indicating that the SBA-15 was successfully silanized by 3 -aminopropyl trimethoxysilane. ${ }^{22}$ 


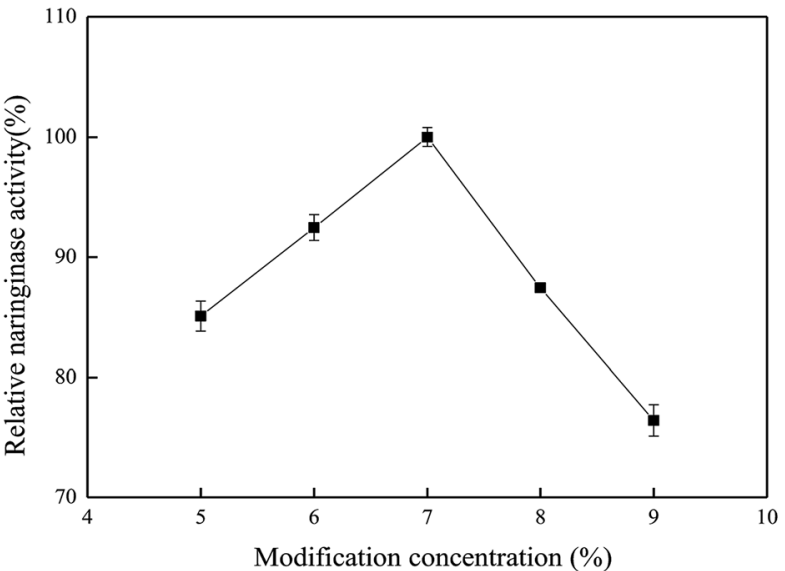

(A)

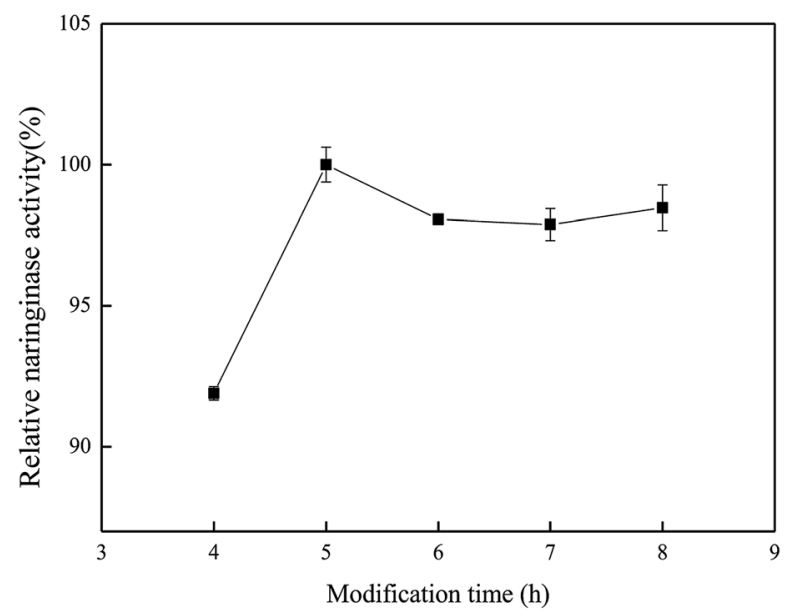

(B)

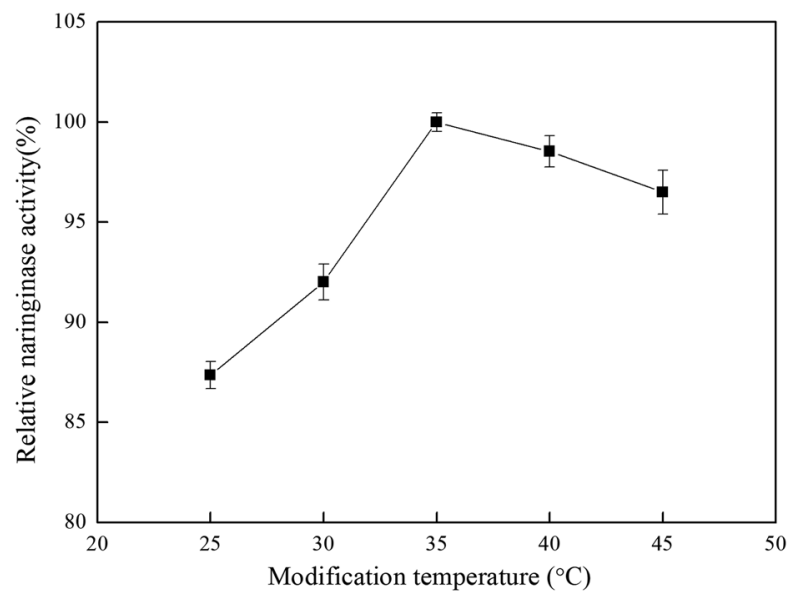

(C)

Fig. 2 The effects of modification glutaraldehyde concentration (A), modification time (B) and modification temperature (C) on immobilized naringinase activity (the experiments were carried out in triplicate).

The results from nitrogen adsorption/desorption isotherms are shown in Table 1. After silanization, the specific surface of SBA-15- $\mathrm{NH}_{2}$ decreased from $1.286 \mathrm{~cm}^{3} \mathrm{~g}^{-1}$ to $1.066 \mathrm{~cm}^{3} \mathrm{~g}^{-1}$, and the total pore volume decreased $16.7 \%$ (from $448.296 \mathrm{~m}^{2}$ to $373.692 \mathrm{~m}^{2}$ ), which suggested that SBA-15 was successfully silanized. When it came to the glutaraldehyde modification of

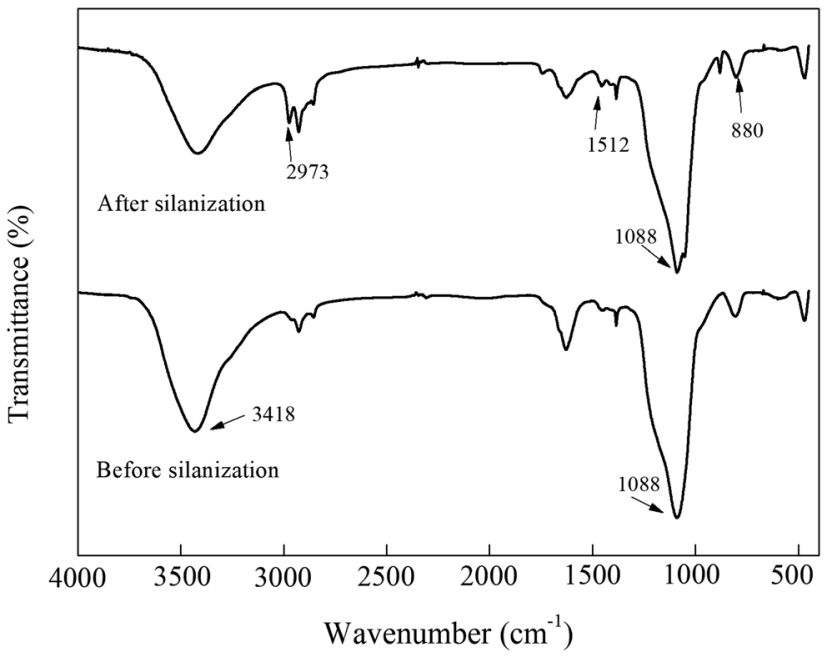

Fig. 3 FT-IR spectra of SBA-15 and silanized SBA-15.

silanized SBA-15, the specific surface of SBA-15-CHO decreased to $7.577 \times 10^{-1} \mathrm{~cm}^{3} \mathrm{~g}^{-1}$, and the total pore volume further decreased $16.5 \%$ (from $373.692 \mathrm{~m}^{2}$ to $312.092 \mathrm{~m}^{2}$ ). This indicated that glutaraldehyde reacted with aminogroups on the surface or in the pores of the silanized SBA15. Finally, the specific surface of the immobilized naringinase was $5.608 \times 10^{-1} \mathrm{~cm}^{3} \mathrm{~g}^{-1}$, and the total pore volume was $273.736 \mathrm{~m}^{2}$, both of which were the lowest values compared with the others, illustrating that the naringinase was successfully linked to the surface or in the pores of the material.

\subsection{Optimization of immobilization parameters}

3.3.1 Effect of immobilization temperature. As can be seen from Fig. 4(A), the optimized immobilization temperature was $35{ }^{\circ} \mathrm{C}$, with an enzyme activity of $423.70 \mathrm{U} \mathrm{g}^{-1}$. The activity of immobilized naringinase increased as the temperature increased from $25^{\circ} \mathrm{C}$ to $35^{\circ} \mathrm{C}$, and decreased as the temperature increased from $35{ }^{\circ} \mathrm{C}$ to $45{ }^{\circ} \mathrm{C}$, which might be due to the temperature sensitivity of naringinase. Further experiment indicated that when the immobilization temperature exceeded $65{ }^{\circ} \mathrm{C}$, the naringinase activity decreased significantly.

3.3.2 Effect of immobilization pH. During the immobilization, the $\mathrm{pH}$ of the fermentation broth greatly influenced the immobilized naringinase activity. In Fig. 4(B), it is seen that immobilized naringinase had good acid resistance. It could maintain more than $88 \%$ relative naringinase activity in the acidic environment, and at $\mathrm{pH} 3.5$ the immobilized naringinase

Table 1 The specific surface area and total pore volume of materials at different modification stages

\begin{tabular}{lll}
\hline Sample & $\begin{array}{l}\text { Specific surface } \\
\text { area }\left(\mathrm{cm}^{3} \mathrm{~g}^{-1}\right)\end{array}$ & $\begin{array}{l}\text { Total pore volume } \\
\left(\mathrm{m}^{2}\right)\end{array}$ \\
\hline SBA-15 & 1.286 & 448.296 \\
SBA-15-NH & 1.066 & 373.692 \\
SBA-15-CHO & $7.577 \times 10^{-1}$ & 312.092 \\
Immobilized naringinase & $5.608 \times 10^{-1}$ & 273.736
\end{tabular}




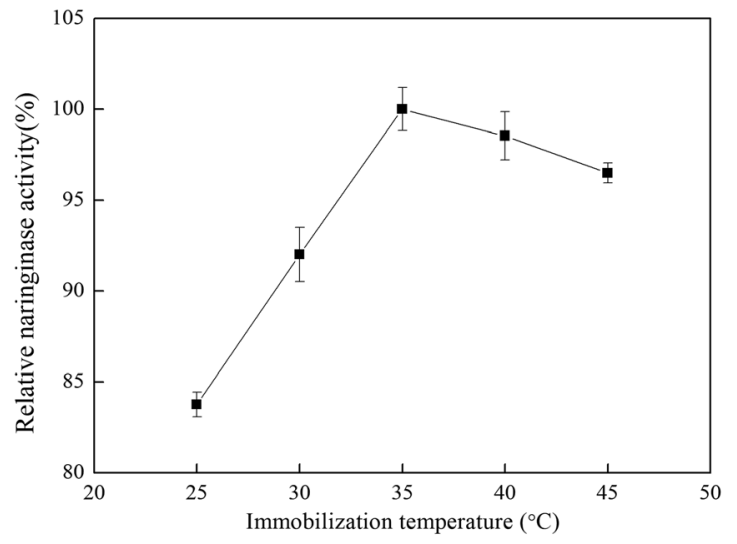

(A)

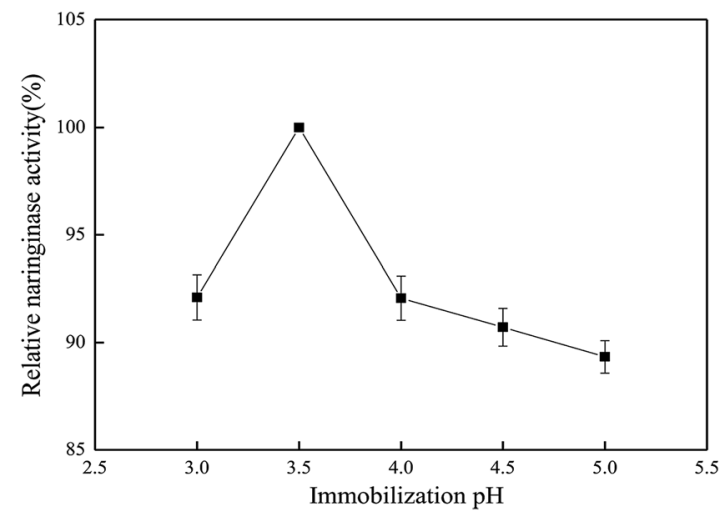

(B)

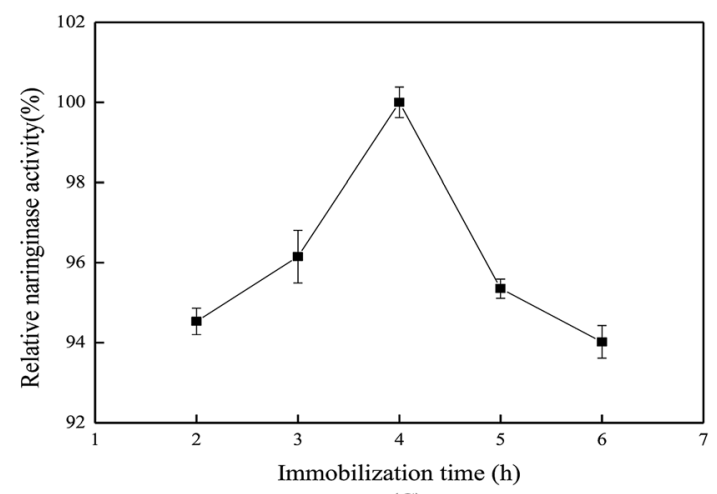

(C)

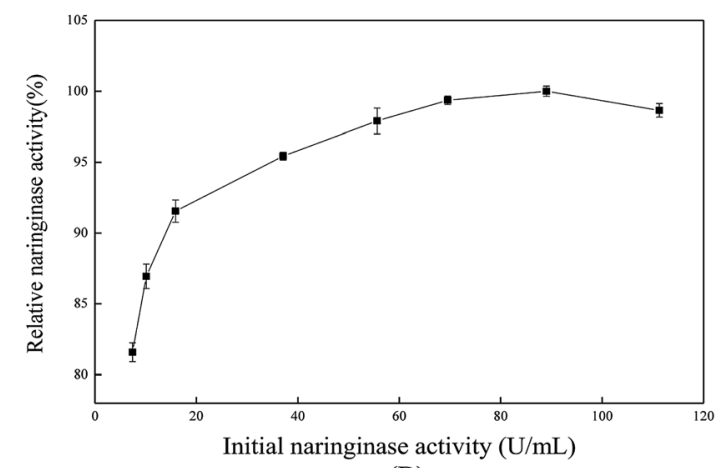

(D)

Fig. 4 The effects of immobilization temperature (A), immobilization $\mathrm{pH}(\mathrm{B})$, immobilization time (C) and initial naringinase activity (D) on immobilized naringinase activity (the experiments were carried out in triplicate). showed the highest naringinase activity. This was because the $\mathrm{pH}$ value affected the ionization of certain groups in the protein. At different $\mathrm{pH}$ values, the naringinase molecules were not in the same state, resulting in the different naringinase activities. Furthermore, the surface of SBA-15 had negative charge, while naringinase contained residual positive charge, which made the naringinase easily immobilized in acidic conditions. ${ }^{23}$

3.3.3 Effect of immobilization time. The effect of immobilization time on immobilized naringinase activity is shown in Fig. 4(C), and the optimum immobilization time was $4 \mathrm{~h}$. It was demonstrated that when the immobilization time was within the range $2-4 \mathrm{~h}$, the relative naringinase activity increased. As SBA-15 had a porous structure, during the naringinase catalysis reaction the molecular diffusion within the material took some time. Therefore, free naringinase required sufficient time to bind to the material.

When the immobilization time reached $4 \mathrm{~h}$, the immobilized naringinase exhibited the highest activity. However, at immobilization times longer than $4 \mathrm{~h}$, the relative naringinase activity decreased rapidly. That might because when the time reached $4 \mathrm{~h}$, the amount of immobilized naringinase reached saturation, and longer immobilization times caused more naringinase to attach to the surface or in the pores of the material, which increased the steric hindrance effect between the naringinase molecules, and also covered the active centers of the naringinase. As a result, the naringinase activity finally decreased. ${ }^{24}$

3.3.4 Effect of the initial naringinase activity. As shown in Fig. 4(D), the immobilized naringinase activity increased with the increase of initial naringinase activity. When the initial naringinase activity was $89.04 \mathrm{U} \mathrm{mL}^{-1}$, the immobilized naringinase activity reached the highest value, and for further increases of initial naringinase activity the immobilized naringinase activity was stable. Compared with Diab et $a .^{25}$ and Duan et al. ${ }^{26}$, in this work, it was indicated that the immobilized naringinase had higher naringinase activity.

As discussed above, the optimized parameters for naringinase immobilization were: temperature $35^{\circ} \mathrm{C}, \mathrm{pH}$ value 3.5 , time $4 \mathrm{~h}$, initial naringinase concentration $89.04 \mathrm{U} \mathrm{mL}^{-1}$; the immobilized naringinase-carrying rate, naringinase activity recovery and naringinase activity were evaluated as $63.66 \%, 87.64 \%$ and $467.62 \mathrm{U}$ $\mathrm{g}^{-1}$, respectively. Compared with previous literature, ${ }^{27}$ the immobilized naringinase activity in this work was higher.

\subsection{Hydrolysis properties of the immobilized naringinase}

3.4.1 The optimum reaction conditions of immobilized naringinase. From Fig. 5(A) and (B), the optimized parameters of naringin hydrolysis by the immobilized naringinase were determined to be reaction temperature of $45^{\circ} \mathrm{C}$ and $\mathrm{pH}$ at 4.5 , whereas those of free naringinase were $50{ }^{\circ} \mathrm{C}$ and 4.5 , respectively. As shown in Fig. 5(A), the immobilized naringinase activity fluctuated slightly with the change of temperature. It was also indicated that immobilization could remarkably improve the temperature stability and acid resistance of naringinase. The SBA-15 immobilized naringinase had a wide $\mathrm{pH}$ range and excellent thermal stability, which was similar to the experimental results of $\mathrm{Xu}$ et al. and Lei et al. ${ }^{28,29}$ Under the 


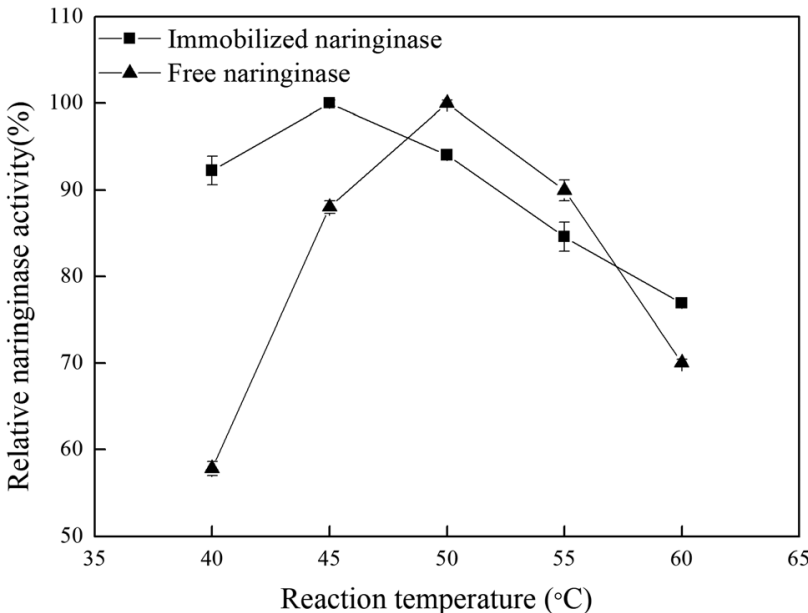

(A)

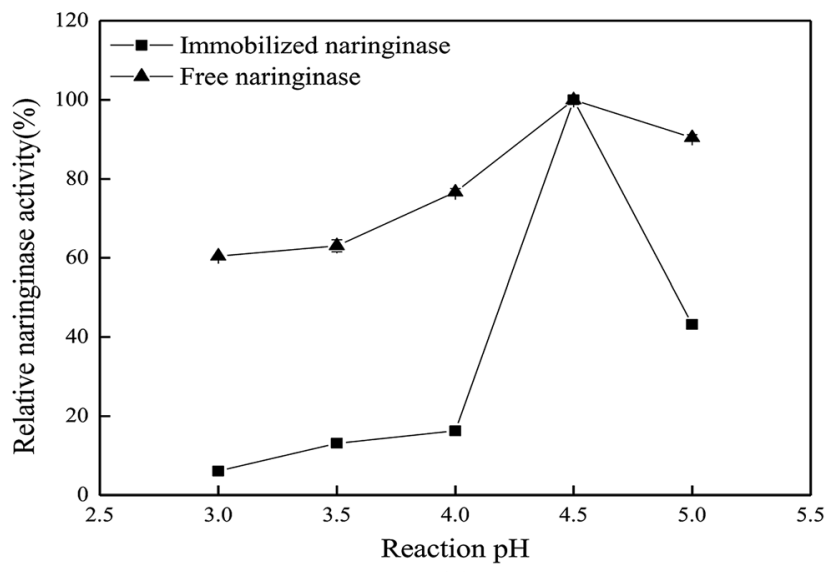

(B)

Fig. 5 The effects of reaction temperature $(A)$ and reaction $\mathrm{pH}(\mathrm{B})$ on free naringinase and immobilized naringinase activity (the experiments were carried out in triplicate).

optimal reaction conditions, the activity of immobilized naringinase reached $517.43 \mathrm{U} \mathrm{g}^{-1}$.

3.4.2 Recycling of immobilized naringinase. Immobilized naringinase was prepared using the optimal immobilization conditions and then was utilized for the reusability experiment. Fig. 6 shows the recycling properties of the immobilized naringinase, indicating that the immobilized naringinase retained $61.81 \%$ of its initial enzymatic activity after being reused eight times. The operational stability of the SBA-15 immobilized naringinase was basically similar to that found by Devesh et al. ${ }^{30}$ Naringinase immobilized on SBA-15 has good operational stability, which brings great development potential in naringinase application for debittering of fruit juice and product quality improvement.

3.4.3 Storage stability of immobilized naringinase. The storage stability of immobilized naringinase and free naringinase is shown in Fig. 7, indicating that immobilized naringinase retained more than $80 \%$ relative naringinase activity after 30 days of storage. In contrast, the activity of free naringinase was significantly decreased during the storage period (less than $30 \%$ relative naringinase activity). The results brought out that the immobilized naringinase had excellent

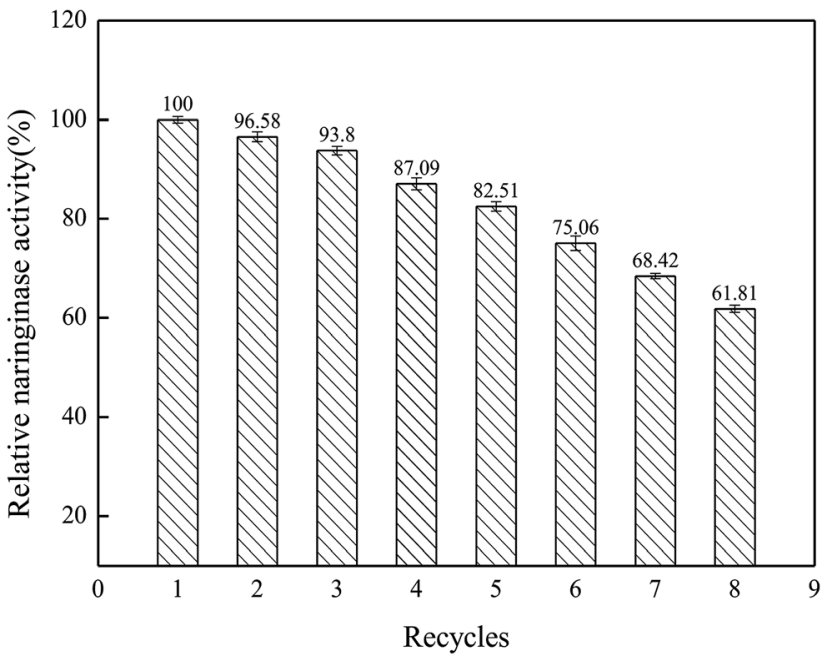

Fig. 6 Recycling of immobilized naringinase (the experiments were carried out in triplicate).

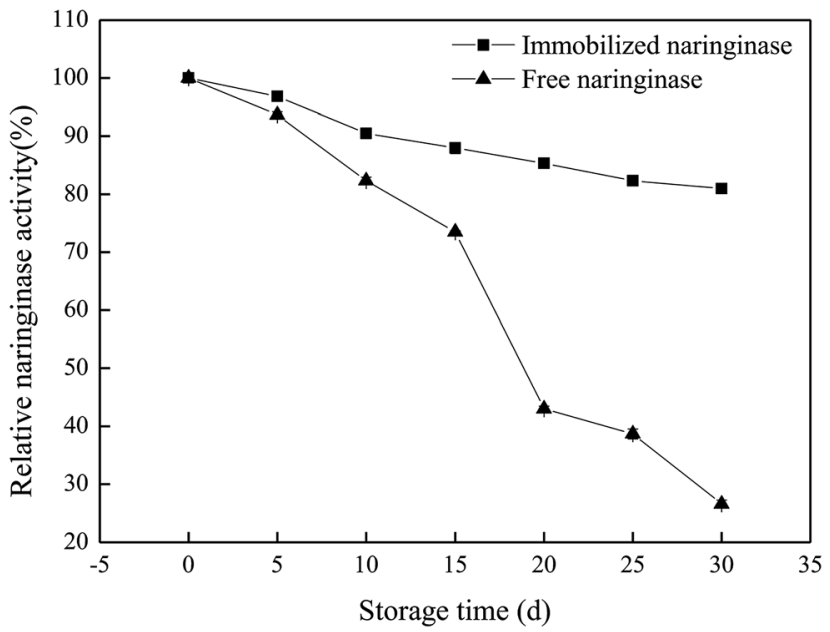

Fig. 7 Storage stability of free naringinase and immobilized naringinase (the experiments were carried out in triplicate).

application potential, especially when the enzyme needed long distance transportation and storage after production.

\section{Conclusions}

In this work, the characteristics and hydrolysis properties of immobilized naringinase on porous silicon material were studied. SBA-15 was suitable for the immobilization of naringinase after silanization and modification, and the optimum conditions for the naringinase immobilization were an initial naringinase activity of $89.04 \mathrm{U} \mathrm{mL}^{-1}$, temperature of $40{ }^{\circ} \mathrm{C}, \mathrm{pH}$ of 3.5 and refrigerated storage time of $4 \mathrm{~h}$, and the highest naringinase activity achieved was $517.43 \mathrm{U} \mathrm{g}^{-1}$. Additionally, the immobilized naringinase had excellent operational stability, and retained $61.81 \%$ of its enzymatic activity after being reused 8 times. Furthermore, after 30 days of storage at $4{ }^{\circ} \mathrm{C}$, it still retained $80.95 \%$ of the naringinase activity. Therefore, as a novel enzyme immobilization material, SBA-15 would enable 
great progress to be made in the application of naringinase and the development of the fruit juice industry.

\section{Conflicts of interest}

There are no conflicts to declare.

\section{Acknowledgements}

This work was supported by the National Natural Science Foundation of China (No. 31601411 \& No. 21804129). We are grateful for the test analysis support from the Analysis Center of Dalian Polytechnic University and Dalian Institute of Chemical Physics, Chinese Academy of Sciences.

\section{References}

1 G. Şekeroğlu, S. Fadıloğlu and F. Göğüss, Immobilization and characterization of naringinase for the hydrolysis of naringin, Eur. Food Res. Technol., 2006, 224(1), 55-60.

2 P. Chine, F. U. Shen and T. Yuan, Monitoring enzymatic debittering in grapefruit juice by high performance liquid chromatography, J. Food Drug Anal., 2001, 9(2), 115-120.

3 Daiichi Sankyo Company Limited, Preparation of antibiotic chloropolysporin-C, Japanese Patent, 63146797, 1988.

4 J. Beekwilder, D. Marcozzi, S. Vecchi, et al., Characterization of rhamnosidases from Lactobacillus plantarum and Lactobacillus acidophilus, Appl. Environ. Microbiol., 2009, 75(11), 3447-3454.

5 M. H. Ribeiro, Naringinases: occurrence, characteristics, and applications, Appl. Microbiol. Biotechnol., 2011, 90(6), 18831895.

6 A. K. Bajpai and S. Bhanu, Immobilization of $\alpha$-amylase in vinyl-polymer-based interpenetrating polymer networks, Colloid Polym. Sci., 2003, 282(1), 76-83.

7 C. Li, Q. Zhang, Y. Wang and H. Wan, Preparation, characterization and catalytic activity of palladium nanoparticles encapsulated in SBA-15, Catal. Lett., 2008, 120, 126-136.

8 X. Fang, Y. Cao, W. Liang, et al., Conventional treatment technology and silanization technology for the surface of magnesium alloy, J. Chongqing Univ. Sci. Technol., Nat. Sci. Ed., 2010, 2, 123-125.

9 M. Lian, Q. Li, J. Tian, et al., Process optimization of naringinase production improved by fungi co-culture, $J$. Chem. Eng. Chin. Univ., 2017, 31(2), 394-400.

10 S. Wei, Q. Wang, J. Zhu, et al., Multifunctional composite core-shell nanoparticles, Nanoscale, 2011, 3(11), 4474-4502.

11 C. T. Kresge, M. E. Leonowicz, W. J. Roth, et al., Ordered mesoporous molecular sieves synthesized by a liquidcrystal template mechanism, Nature, 1992, 359(6397), 710712.

12 C. W. Kwong, C. Y. H. Chao, K. S. Hui, et al., Catalytic ozonation of toluene using zeolite and MCM-41 materials, Environ. Sci. Technol., 2015, 42(22), 8504-8509.
13 D. Y. Zhao, J. L. Feng, Q. S. Huo, et al., Triblock copolymer syntheses of mesoporous silica with periodic 50 to 300 angstrom pores, Science, 1998, 79, 548-552.

14 M. Tao, Z. Xin, X. Meng, et al., Highly dispersed nickel within mesochannels of SBA-15 for CO methanation with enhanced activity and excellent thermostability, Fuel, 2017, 188, 267-276.

15 A. J. Shen, Development of novel hydrophilic interaction liquid chromatographic stationary phases based on thiol-ene click chemistry, University of Chinese Academy of Sciences, 2015.

16 W. H. Zhang, X. B. Lu, J. H. Xiu, et al., Synthesis and characterization of bifunctionalized ordered mesoporous materials, Adv. Funct. Mater., 2004, 14(14), 544-552.

17 W. Zeng, X. F. Qian, Y. B. Zhang, et al., Organic modified mesoporous MCM-41 through solvothermal process as drug delivery system, Mater. Res. Bull., 2005, 40(5), 766-772.

18 I. Safarik, K. Pospisk, Z. Mader, et al., Microwave-synthesized magnetic chitosn microparticles for the immobilization of yeast cells, Yeast, 2015, 32(1), 239-243.

19 L. F. Zhao, Study on the immobilization of lipase and its catalytic performances, Jilin University, 2008.

20 W. B. Davis, Determination of flavanones in citrus fruits, Anal. Chem., 1947, 19(7), 476-478.

21 P. W. Cui, J. Li, Z. Xiao, et al., Immobilization of Penicillium sp. naringinase on epoxy resin, Food Ferment. Ind., 2014, 40(5), 87-92.

22 Y. M. Wang and H. Yuan, Separation and preconcentration of trace cadmium(II) in water with aminopropyl silatrane immobilized on mesoporous silica, J. Instrum. Anal., 2015, 34(12), 1382-1386.

23 H. Takahashi, B. Li, T. Sasaki, et al., Immobilized enzymes in ordered mesoporous silica materials and improvement of their stability and catalytic activity in an organic solvent, Microporous Mesoporous Mater., 2001, 44(45), 755-762.

24 B. Hu, J. Pan, H. L. Yu, et al., Immobilization of Serratia marcescens lipase onto amino-functionalized magnetic nanoparticles for repeated use in enzymatic synthesis of Diltiazem intermediate, Process Biochemistry, 2009, 44(9), 1019-1024.

25 M. A. Diab, A. Z. El-Sonbati, M. M. Al-Halawany, et al., Thermal stability and degradation of chitosan modified by cinnamic acid, Open J. Polym. Chem., 2012, 2(1), 14-20.

26 Z. Duan, J. Xia and Y. Shan, Comparative study of different materials immobilized naringinase, J. Food Sci. Technol., 2015, 33(3), 43-48.

27 M. A. P. Nunes, R. F. Vila, et al., Immobilization of naringinase in PVA-alginate matrix using an innovative technique, Appl. Biochem. Biotechnol., 2010, 160(7), 21292147.

28 J. Xu, X. T. Sun, Q. Li, et al., Study on hydrolysis of naringin by immobilized naringinase on magnetic silica chitosan microspheres, Jingxi Huagong, 2018, 35(11), 1885-1892.

29 S. J. Lei, X. K. Wang, et al., Immobilization of naringinase on PVA-calcium alginate gel, Food Science, 2011, 32(3), 138-143.

30 K. Devesh, T. Mahe, O. N. Srivastava, et al., Immobilization of $\beta$-galactosidase onto functionalized graphene nanosheets using response surface methodology and its analytical appli-cations, PLoS One, 2012, 7(7), 1-13. 